\title{
Non-Literal Word Sense Identification Through Semantic Network Path Schemata
}

\author{
Eric Iverson, Stephen Helmreich \\ Computing Research Lab and Computer Science Department \\ Box 30001/3CRL \\ New Mexico State University \\ Las Cruces, NM 88003-0001
}

When computer programs disambiguate words in a sentence, they often encounter non-literal $\alpha$ novel usages not included in their lexicon. In a recent study, Georgia Green (personal communication) estimated that $17 \%$ to $20 \%$ of the content word senses encountered in various types of normal English text are not listed in the dictionary. While these novel word senses are generally valid, they occur in such great numbers, and with such little individual frequency that it is impractical to explicitly include them all within the lexicon. Instead. mechanisms are needed which can derive novel senses from existing ones; thus allowing a program to recognize a significant set of potential word senses while keeping its lexicon within a reasonable size.

Spreading activation is a mechanism that allows us to do this. Here the program follows paths from existing word senses stored in a semantic network to other closely associated word senses. By examining the shape of the resultant path, we can determine the relationship between the senses contained in the path; thus deriving novel composite meanings not contained within any of the original lexical entries. This process is similar to the spreading activation and marker passing techniques of Hirst [1988], Charniak [1986], and Norvig [1989] and is embodied in the Prolog program metallel based on Fass' program meta5 (Fass [1988]).

Metallel's lexicon is written as a series of sense frames, each containing information about a particular word sense. A sense frame can be broken into two main parts: genera and differentiae. Genera are the genus terms that function as the ancestors of a word sense. Differentiae denote the qualities that distinguish a particular sense from other senses of the same genus. Differentiae can be broken down into source and target which hold, respectively, the preferences $^{1}$ and properties of a sense. Source contains differentiae information concerning another word sense. Target information concems the sense itself.

Connections can be found to other word senses in one of two ways: through an ancestor relationship (genus) or through a preference or property relationship (differentia). In the case of differentiae, it is necessary to extract the word senses from a higher order structure. For example, [it $(n, 1)$, contain $(v, 1)$, music $(n, 1)]$ is not a word sense that is listed in the lexicon, while music $(n, 1)$ is listed. It is therefore necessary to extract music $(n, 1)$ from the larger differentia structure in which it occurs and add it to the path.

Not all paths are valid, indicating that some criteria of acceptability are needed during analysis. In addition, paths that are superficially different often end up being quite similar upon further analysis. Keeping this in mind, we have attempted to identify path schemata and associate them with types of nonliteral usage. Specifically, we have concentrated on identifying instances of metaphor and metonymy.

A metaphorical path schema is one in which the preference of a verb and the actual target of the preference both reference different 3 place differentiae $^{2}$ which can be said to be related. Two 3 place

\footnotetext{
1 Preferences indicate the semantic category of the word sense that fills a specific semantic role with respect to the word sense being defined. For example, the transitive sense of the verb eat prefers (in normal usage) an animate subject and an edible object. Violations of these preferences are indications of non-literal usage. (See Wilks and Fass [1990].)

${ }^{2}$ A 3 place differentia is a list of senses following a [Subject, Verb, Object] format in which either the Subject or the Object consists of the pronominal token it $(n, 1)$.
} 
differentiae are related if both their respective subjects and objects are identical or form a "sister" relationship ${ }^{3}$. Additionally, the two verbs of the differentiae as well as the verb which generated the preference must have a similar relationship

The ship ploughed the waves.

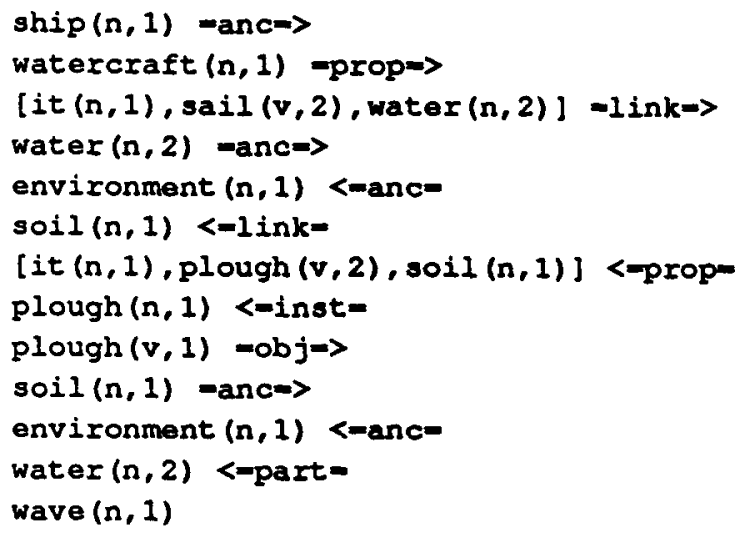

For example in the path for the sentence The ship ploughed the waves, [it $(n, 1)$, sail $(v, 2)$, water $(n, 1)]$ and $[i t(n, 1)$, plough $(v, 2)$, soil $(n, 1)]$ are related since plough $(v, 1)$, plough $(v, 2)$ and sail $(v, 2)$ are children of transfer $(v, 1)$, and water $(n, 1)$ and soil $(n, 1)$ are children of environment $(n, 1)$. Also, the pivot nodes ${ }^{4}$ for the instrument and object preferences of plough $(v, 1)$ are both environment $(n, 1)$, thereby indicating an even stronger relationship between the instrument and the object of the sentence. Thus, an analogy exists between ploughing soil and sailing water, suggesting a new sense of plough that combines aspects of both.

Denise drank the bottle.

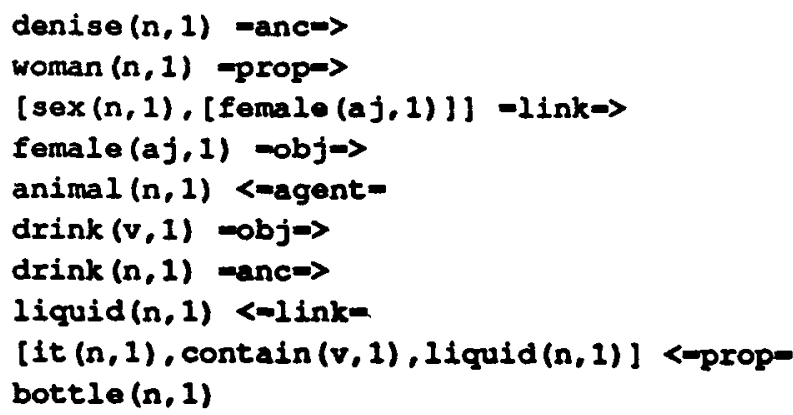

3 Two word senses are sisters if they have a close com. mon ancestar.

A pivot node is a node with two incoming edges.
A metonymic path is indicated when a path is found from a target sense through one of its inherited differentiae; thus linking the original sense to a related sense through a property or preference relationship. For example in the sentence Denise drank the bottle, one of the properties of bottle $(n, 1)$ is $[$ it $(n, 1)$, contain $(v, 1)$, liquid $(n, 1)]$. This differentia allows us to derive a novel metonymic word sense for bottle in which the bottle's contents are denoted rather than the bottle itself. Under metallel, any differentia can act as a conduit for a metonymy; thus facilitating the generation of novel metonymies as well as novel word senses.

By using semantic network path schemata to identify instances of non-literal usage, we have expanded the power of our program without doing so at the expense of a larger lexicon. In addition, by keeping our semantic relationship and path schema criteria at a general level, we hope to be able to cover a wide variety of different semantic taxonomies.

\section{References}

Charniak, E. 1986. A neat theory of marker passing. Procs. AAAI-86. Philadelphia, PA.

Fass, D. 1988. Collative Semantics: A Semantics for Natural Language Processing. Memoranda in Computer and Cognitive Science, MCCS88-118. Computing Research Laboratory, New Mexico State University.

Hirst, G. 1988. Resolving lexical ambiguity computationally with spreading activation and polaroid words. In Small and Cottrell (eds.), Lexical Ambiguity Resolution pp. 73-107. Morgan Kaufmann: San Mateo.

Norvig, P. 1989. Marker passing as a weak method for text inferencing. Cognitive Science 13(4):569-620.

Wilks, Y., and D. Fass. 1990. Preference Semantics. Memoranda in Computer and Cognitive Science. MCCS-90-194. Computing Research Laboratory, New Mexico State University. 